\title{
Strategy equilibrium in dilemma games with off-diagonal payoff perturbations
}

\author{
Marco A. Amaral $\odot^{1, *}$ and Marco A. Javarone ${ }^{2, \dagger}$ \\ ${ }^{1}$ Instituto de Humanidades, Artes e Ciências, Universidade Federal do Sul da Bahia-BA, 45996-108, Brazil \\ ${ }^{2}$ Department of Mathematics, University College London, London WC1E 6BT, United Kingdom
}

(Received 1 April 2020; accepted 3 June 2020; published 19 June 2020)

\begin{abstract}
We analyze the strategy equilibrium of dilemma games considering a payoff matrix affected by small and random perturbations on the off-diagonal. Notably, a recent work [Proc. R. Soc. A 476, 20200116 (2020)] reported that while cooperation is sustained by perturbations acting on the main diagonal, a less clear scenario emerges when perturbations act on the off-diagonal. Thus, the second case represents the core of this investigation, aimed at completing the description of the effects that payoff perturbations have on the dynamics of evolutionary games. Our results, achieved by analyzing the proposed model under a variety of configurations as different update rules, suggest that off-diagonal perturbations actually constitute a nontrivial form of noise. In particular, the most interesting effects are detected near the phase transition, as perturbations tend to move the strategy distribution towards nonordered states of equilibrium, supporting cooperation when defection is pervading the population, and supporting defection in the opposite case. To conclude, we identified a form of noise that, under controlled conditions, could be used to enhance cooperation and greatly delay its extinction.
\end{abstract}

DOI: 10.1103/PhysRevE.101.062309

\section{INTRODUCTION}

Perturbative methods find large utilization for studying a number of problems in physics, spanning from classical to quantum mechanics [1,2]. Furthermore, as we know from the theory of chaos [3], even small perturbations can have drastic effects on the dynamics of some systems, as those particularly sensible to initial conditions. The logistic map, for instance, is very useful for studying these phenomena. Also, the theory of chaos has the merit to have widely popularized fascinating results, such as the so-called "butterfly effect," being currently mentioned both in books and movies (e.g., [4]), although sometimes without proper scientific care.

Perturbations have also been used in evolutionary game theory for studying the emergence of cooperation [5-7], as recently reported in [8]. Notably, the latter shows that small and random perturbations on the payoff matrix of social dilemmas can strongly influence the strategy equilibrium of a population. However, while their effect on the main diagonal has been clarified, i.e., they support cooperative behaviors, the effect given by off-diagonal perturbations is still unclear. Thus, here we aim to clarify this specific aspect of the perturbative method presented in [8] and to obtain a complete description of the dynamics of evolutionary games, affected by small and random perturbations on their payoff matrix. To this end, our analyses consider a variety of conditions, as three different update rules, and various games (e.g., stag hunt, snowdrift, and harmony). For the sake of clarity and for making the manuscript self-contained, the main model is fully described in the following section, with all relevant information. Before moving further, let us give a brief introduction on

\footnotetext{
*marcoantonio.amaral@gmail.com

†marcojavarone@gmail.com
}

the framework of evolutionary game theory (hereafter EGT) that we use for our investigation.

EGT is a suitable framework to study the emergence of cooperation among selfish individuals [9-11]. While cooperation still represents a lively challenge [12], EGT has given many important insights on how such a phenomenon can spontaneously emerge at many scales in biological and social systems. Among the most studied mechanisms are kin selection [13], direct and indirect reciprocity [14,15], network reciprocity [16-19], group selection [20], and heterogeneity [21-25]. Specifically, heterogeneity (sometimes dubbed as diversity) was found to be a fundamental element for supporting cooperative behaviors in evolutionary systems [26,27]. A group composed of individuals with different skills can easily become more effective in several challenges. At the same time, group diversity can shield the possible flaws of individual members while sustaining the whole group in a synergetic manner. This rationale has been applied to economies, enterprise communities, vaccination models, and even in biological evolution [26,28-31]. It also represents a fundamental aspect in genetic algorithms as well [32], whose core mechanism is based on a heterogeneous population of candidate solutions that evolve towards a (sub)optimal solution. In EGT, heterogeneity can be related to different aspects of a game, e.g., incentives, interaction topology, learning rates, and dynamics. Previous investigations, such as $[22,29,33-38]$, showed that heterogeneity can support cooperative behaviors in many competitive scenarios. At the same time, other studies such as [39] reported that heterogeneous networks do not promote cooperation when humans play the prisoner's dilemma. As another example, findings reported in [40] show a relationship between the group size of a community and its heterogeneity. This rich spectrum of results highlights the need to further study this subject and to fully understand its range of effects. 
As observed in [8], perturbations on the payoff matrix of dilemma games actually are responsible for a form of population heterogeneity, as they provide players with a diversified perception of risks and rewards. For this reason our investigation, while focused on clarifying the role of perturbations in social dilemmas, can provide findings of potential interest for the debate on the relationships between heterogeneity and cooperation in evolutionary games.

Also, the relevance of studying the effects of a heterogeneous risk perception is particularly motivated by a simple fact of everyday life, e.g., individuals facing the same situation will have different risk perceptions [11,41,42]. EGT's mathematical framework has proven to be invaluable to characterize the general aspects of decision-making conflicts, creating general game classes to do so. Nevertheless, a core concept of such game classes, the perceived payoff, is still a subjective quantity, one that cannot be directly measured in most contexts (with the obvious exception of totally rational financial trades). In this sense, understanding the core aspects of a system with fluctuating perceived payoffs could lead to the understanding of general behaviors even when the exact perceived payoffs are unknown. This is the usual case of animal conflicts, where a fixed number cannot always be obtained for the perceived payoff but should be expected to fluctuate around an average value. But even in sociophysics this concept is useful, since a common critique of game theory (and sociophysics) is that the diversity and subjectivity of the perceived rewards and risks cannot be described by fixed values of the payoff $[6,7]$. Therefore a framework that allows us to explore general aspects of a "fuzzy" payoff environment could further support the strength of game theory in modeling real-world scenarios.

Financial trading [43], emergency medicine [44], and even poker games [45] are just a few examples we can mention to appreciate the relevance of a heterogeneity in risk and reward perception in a system. For instance, rational but risky decisions can be essential to achieve favorable outcomes, and individuals actually show a variety of behaviors in the above listed activities. The generality of these considerations makes the payoff values suitable for being considered as stochastic variables instead as fixed values (as per the classic EGT approach).

One way to study the influence of such random variations is to represent each different environmental condition as a new factor in the equations of a model. Following this method, many authors have made important advances in the understanding of how a number of conditions can drive the system dynamics, such as resource heterogeneity [46], different behaviors [47,48], seasonal variations [33], diverse learning rates [49], different death rates [50], interaction topologies [51], and so on. However, another way to understand these phenomena is to study the behavior of a population whose evolution can be affected by a payoff matrix constantly perturbed by stochastic noise with zero mean value [52-54], regardless of its origin. In other words, as the environmental perturbations are very diverse and frequent, we can suppose that the sum of infinitely many small perturbations acts as a stochastic perturbation around an average value. Indeed, the central limit theorem indicates that the sum of all these uncorrelated perturbations would probably behave as a Gaussian noise. In this scenario, we are not interested in every single source of perturbation, but rather, in their collective effect.

We deem it relevant to mention previous seminal works, such as [55-59], that (to the best of our knowledge) first studied such disorder in EGT. More recently, the effect of payoff noise on phase transitions has been the core of much research that has shed light on how different types of perturbations can lead to the emergence of cooperation $[8,27,33,52,53,60-$ 66]. So, here, starting from the model proposed in [8], which introduces payoff perturbation in the context of the imitation update rule for all payoff entries, we perform a full analysis under different settings, and then we study the microscopical mechanism that leads to the observed macroscopic results.

After introducing the proposed model in Sec. II, we show the results of numerical simulations (i.e., Sec. III) and, eventually, in Sec. IV we discuss the main findings and their possible implications.

\section{MODEL}

For the sake of simplicity, we consider two-strategy games where players can either cooperate (C) or defect (D). Mutual cooperation yields a payoff $R$ (reward), and mutual defection yields $P$ (punishment). A defector receives a payoff equal to $T$ (temptation) when interacting with a cooperator that in turn receives a payoff equal to $S$ (which usually stands for sucker's payoff). We then introduce the effects of small payoff perturbations, say $\varepsilon$, randomly occurring on the off-diagonal elements of the payoff interaction matrix. As a result, the payoff matrix of any two-player interaction can be written as follows:

$$
\mathrm{C} \quad\left[\begin{array}{cc}
\mathrm{C} & \mathrm{D} \\
R & S+\varepsilon_{S} \\
T+\varepsilon_{T} & P
\end{array}\right],
$$

where $T \in[0,2]$ and $S \in[-1,1]$. Without loss of generality, we set $R=1$ and $P=0$. A similar approach was also introduced in [56]. It is worth clarifying that $\varepsilon_{T}$ and $\varepsilon_{S}$ are independent random variables with zero average value $(\bar{\varepsilon}=$ 0 ), drawn from a uniform distribution unless stated otherwise. At each new player interaction, the fluctuation is randomly drawn again, meaning that the noise is not cumulative nor fixed in time or space. This is similar to the annealed disorder case of condensed matter physics [64,67].

Here, the control parameter for the proposed model is the perturbation strength $D$, where for a uniform distribution we set $-D<\varepsilon_{T}<D$ and $-D<\varepsilon_{S}<D$. It is useful to remark that this payoff matrix parametrization spans four different classes of games in the $\{T, S\}$ parameter space: prisoner's dilemma (PD), snowdrift (SD), stag hunt (SH), and harmony games (HG) [21,51]—see Fig. 1. We emphasize that even if the average value of the perturbation is zero, it can be able to (locally) change the game class to a more friendly or more competitive environment from time to time. However, in the long run, there should be, on average, no unilateral contribution to either.

Before moving to further details, let us briefly emphasize that game theory offers a wide set of games, each one devised to represent a specific scenario. As we can see from the payoff 


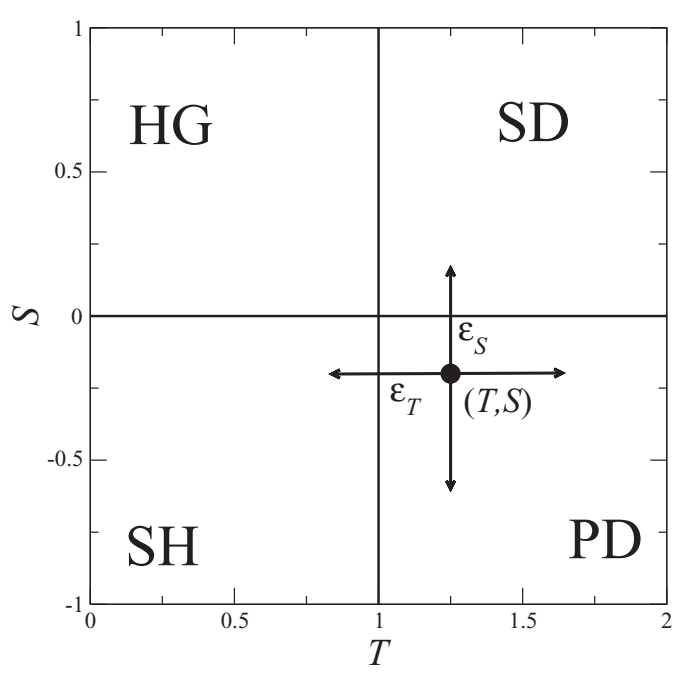

FIG. 1. $T \times S$ parameter space with $R=1, P=0$, spanning four classes of games: prisoner's dilemma (PD), snowdrift (SD), stag hunt $(\mathrm{SH})$, and harmony games (HG). The payoff fluctuation acts over $T$ and $S$ simultaneously and uncorrelated by means of the two independent small random variables, $\varepsilon_{T}$ and $\varepsilon_{S}$. Note that local fluctuations can lead players to play different classes of games depending on the fluctuation strength.

matrix, HG and PD are at the antipodes. In the former, the sucker's and temptation payoff have an overlapping range, so HG has a much higher probability to present cooperation than PD. This reflects the fact that, in the original scenario, two PD players have a tendency to betray each other, and cooperation only results from a substantial risk they would both have to take. On the other hand, SH represents a conflict between safety and social cooperation, while SD, also known as game of chicken or hawk-dove, represents a form of competition where one player succeeds as soon as the opponent gives up (e.g., because of the risk of playing the game itself).

A general core aspect of evolutionary games is given by the strategy update dynamics. We can understand it as a twostep process where: (1) players interact with their neighbors accumulating a payoff, then (2) they may change strategy according to an update rule. Such rules can be defined by taking into account different aspects of any given system. Here we consider the usual imitative rule [51], the Ising (or Glauber dynamics) rule [68], and the dynamic win-stay-loseshift (WSLS) rule [69]. This is done so we can study the robustness of the effects created by payoff perturbation, since previous works have extensively shown how the update rules can lead to different behaviors [22,34,35,47,70,71].

We first present the most usual update rule, the imitative dynamic. Accordingly, one player, say $i$, updates its strategy by comparing its payoff with that of one randomly chosen neighbor, say $j$. Player $i$ adopts the strategy of player $j$ with probability

$$
p\left(\Delta u_{i j}\right)=\frac{1}{1+e^{-\left(u_{j}-u_{i}\right) / k}},
$$

where $k$ is the irrationality level in the decision process [51] (similar to the temperature in statistical physics), while $u_{i}$ and $u_{j}$ represent player $i$ 's and $j$ 's payoff. We use $k=0.1$ for all simulations unless stated otherwise. The imitation rule is a noninnovative dynamic [10,51], because a player can only change its strategy by selecting among those available in the population. This is one of the most explored rules in EGT, and it is associated with the replicator dynamics observed in biological evolution [9]. Because of the extensive research on this setup, we will focus our analysis mainly on this model.

We present next the so-called Ising rule (also known as Glauber dynamics [72]), where players change strategy with probability

$$
p\left(\Delta u_{i}\right)=\frac{1}{1+e^{-\left(u_{i}^{*}-u_{i}\right) / k}},
$$

with $u_{i}$ player $i$ 's payoff, and $u_{i}^{*}$ its potential payoff at a next iteration if it changed strategy while everything else (i.e., the strategy set of its neighborhood) remained the same. Recently, the Ising rule has been studied in $[35,68,73]$, showing that it leads to very different dynamics when compared to imitation models. In mathematical terms, this setting is equivalent to a Monte Carlo protocol used in condensed matter physics to generate ensembles of spin systems that are based on Ising or more general Heisenberg Hamiltonians [74]. In the context of EGT, this update rule is regarded as a player asking himself what would be the benefits of changing its strategy to a different one. This is closely related to rational analysis of a situation (see also [75] on this topic) instead of a reproduction of the "fittest" behavior.

Lastly, we also implement the win-stay-lose-shift update rule with dynamic aspiration [69]. The WSLS strategy relies on cognitive capabilities instead of replicating process [76-79]. In this case, players change strategy depending on the degree of satisfaction with their current payoff in comparison to the average payoff of their neighborhood. The probability of a chosen player to change its strategy to the opposite one is given by

$$
p\left(\Delta u_{i}\right)=\frac{1}{1+e^{-\left(\bar{u}-u_{i}\right) / k}},
$$

where $\bar{u}$ is the average payoff of player $i$ 's neighborhood.

To implement such dynamics we use an asynchronous Monte Carlo protocol so that a random player, say the $i$ th, is selected and its cumulative payoff, as well as those of its firstand second-degree neighbors, are calculated. Then the $i$ th player can change its strategy according to the defined update rule. One Monte Carlo step (MCS) consists of this process being repeated until each player has had the opportunity to change its strategy (that is, $N$ times). So, we wait for the system to reach a dynamic equilibrium (around $10^{4} \mathrm{MCSs}$ ), and then we average the values over the final 1000 steps. This is repeated for 50-100 different samples with the same parameters. A square lattice, with von Neumann neighborhood and periodic boundary conditions with $N=10^{4}$ individuals, is the background for our simulations. A detailed discussion on Monte Carlo methods in evolutionary dynamics is provided in $[51,80]$.

\section{RESULTS}

In this section we show the results of numerical simulations of the proposed model. For the sake of clarity, irrelevant error 

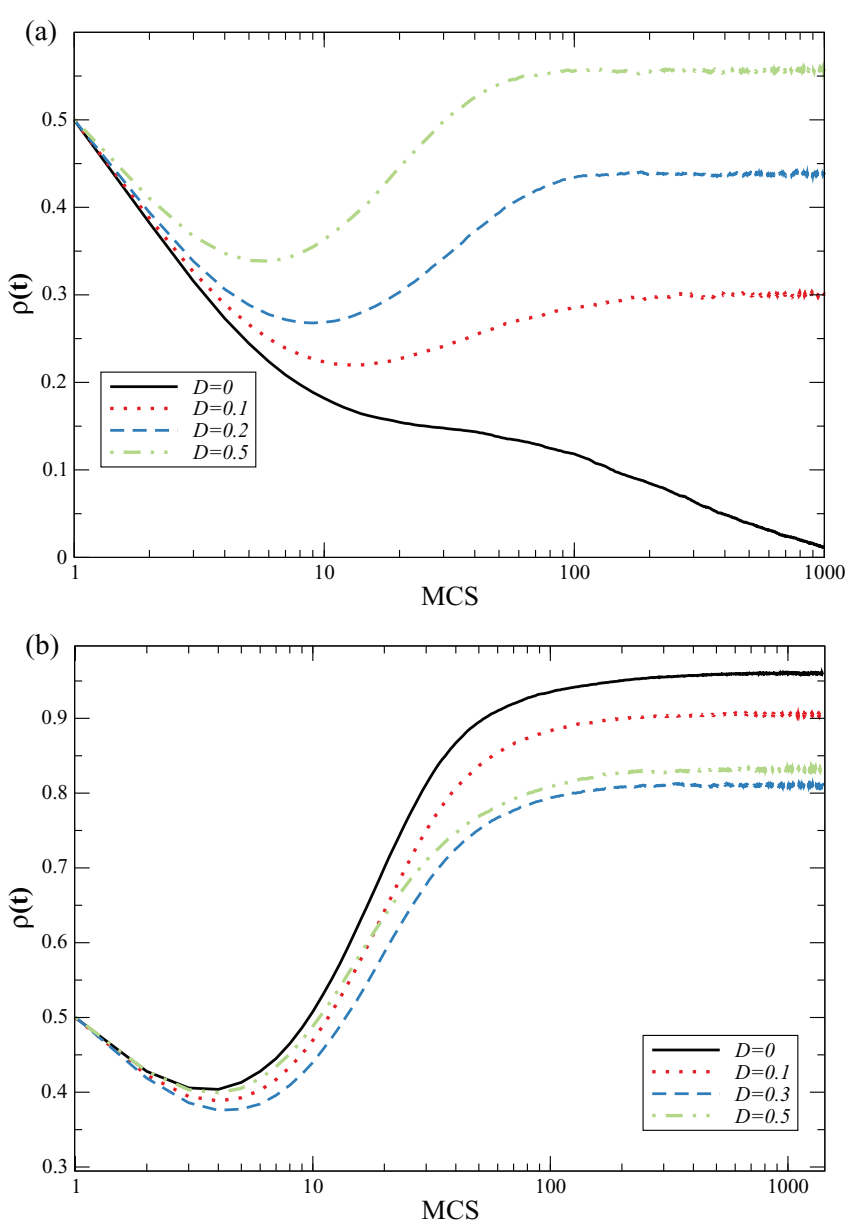

FIG. 2. Average evolution of cooperation, $\rho(t)$, for 100 samples using the imitative update rule. We set $T_{c}=1.04$ in (a), the critical extinction point for cooperation. Even when the noise is around $10 \%$ of the $T$ value, cooperation can reemerge. (b) A similar analysis for $T=0.96$. Notice that the $x$ axis is logarithmic.

bars are hidden. Figure 2(a) presents the average cooperation fraction $\rho(t)$ of a population as time passes, considering the imitative rule. Also, it shows the evolution of cooperation for the parameters $T_{c}=1.04$ and $S=0$. We note that for the imitative model with no payoff perturbation, $T_{c}=1.04$ corresponds to the phase transition point where cooperation becomes extinct. This was chosen to highlight the positive effect of payoff perturbation, since its strongest effect happens near the phase transition boundary. Remarkably, adding a perturbation as low as $D=0.05$ can prevent said extinction, and for $D=0.2$, cooperation can increase even up to $40 \%$. We note that this increase in cooperation also happens for a wide range of $T$ values. Qualitatively similar results were obtained for the Ising and WSLS update rule regarding the temporal evolution of the population.

The analysis of the temporal evolution indicates that the noise almost immediately affects the cooperative behavior. Using the definitions of Tanimoto [64], we can observe that the "endurance" phase (where cooperation initially falls) is drastically affected. Notably, even for $M C S<20$, the noise enhances cooperation, preventing its extinction. The "expanding" phase is therefore reached for very short times, allowing cooperation to flourish. Also note that Fig. 2(b) presents the average temporal behavior for $T=0.96$. We observe that the perturbation can be detrimental to cooperation if $T<1$, that is, for more fraternal games. Note, however, that this detrimental effect is weaker than the positive effect when $T>1$.

Next we focus on the final equilibrium fraction of cooperators $\rho$ for the whole range of $T$. For simplicity, we present results for the weak prisoner's dilemma configuration (i.e., $S=0$ ). We ran simulations for different $S$ values, and the general trends are maintained. Figure 3 presents the outcomes achieved by using the uniform noise distribution in all considered update rules (i.e., imitative, WSLS, and Ising). Its inset shows $\left(\rho-\rho_{0}\right)$, where $\rho_{0}$ is the cooperation value for the case $D=0$. This is especially useful to compare the effects of the payoff perturbation with the unperturbed case, filtering off the effects of varying $T$. It is interesting to note that there is a general trend in all models, i.e., the noise increases cooperation in the PD region $(T>1)$, and such enhancement grows with the noise amplitude $D$. We stress here how the three update rules have very different dynamics and that even so, the general effect of the payoff noise was maintained in all three cases. The inset allows understanding the level of enhancement caused solely by the noise. In particular, the imitative and WSLS rules present a clear peak that grows with $D$ while maintaining its position (relative to $T$ ) regardless of the noise level. It is also very interesting to note that this peak happens in $T_{c}=1.04$ for the imitative model, and after that the positive effect begins to decline. This again reinforces that the benefit of payoff perturbation is most strong near the phase transition, although it is not restricted only to this region. At the same time, for $T<1$, the noise can dampen cooperation in different manners for each case. Using the imitative updating rule, the related drop is very shallow (15\%) and almost independent of $D$. That is similar to results achieved by using the WSLS rule. On the other hand, the Ising rule presents a strong drop in $\left(\rho-\rho_{0}\right)$, which is very dependent on $D$.

Note that there is also another interesting characteristic shared by all three models; in general, there is some specific value of $T$ where all lines cross (in the imitative model this effect is less strong but still visible for $D<0.5$ ). For the imitative and Ising models, this point is $T=1$, while for the WSLS the point occurs near $T \simeq 0.58$.

Considering the parameter space, Fig. 1, we see that the point $T=1, S=0$ is singular since it is exactly the most symmetrical setting concerning the four classes of game. For a rational update rule and an infinite well-mixed population, we can see that the cooperative and defective strategies are identical in terms of payoff (it becomes irrelevant for the player which strategy to chose). As our perturbations are also symmetric, there cannot be any incentive to one strategy or the other, since they are identical. Therefore, perturbations should not change the final state of the system compared to the unperturbed setting, independent of the perturbation strength $D$. This argument takes into account only the payoff matrix and supposes an infinite and well-mixed population. In a spatially distributed model with finite population (and using a Fermi distribution), certain correlations can arise that will change said symmetry. Nevertheless, we see that for the 

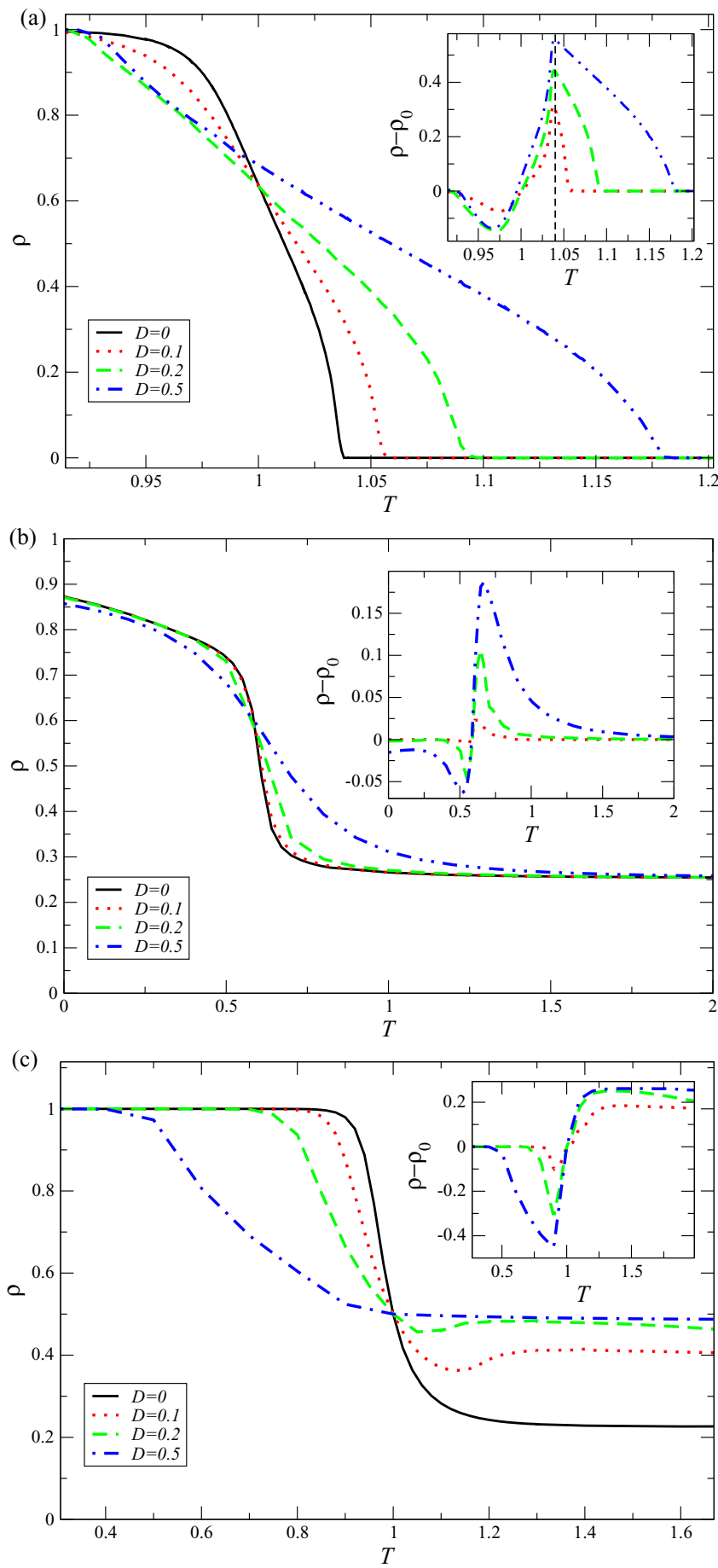

FIG. 3. Average final cooperation level $(\rho)$ as a function of $T$ for the imitative (a), WSLS (b), and Ising (c) models using the uniform noise distribution. The inset shows $\rho-\rho_{0}$, where $\rho_{0}$ is the cooperation value for the case $D=0$.

imitative and Ising model, the point remains near $T=1$ to a high precision. At the same time, the WSLS model does not present this feature for $T=1$, but even so, we can see a very similar behavior in $T$ near 0.58 .

To observe how general the perturbation effect can be, we also analyzed a similar setting but with a Gaussian distribution instead of a uniform one. In this case we set our

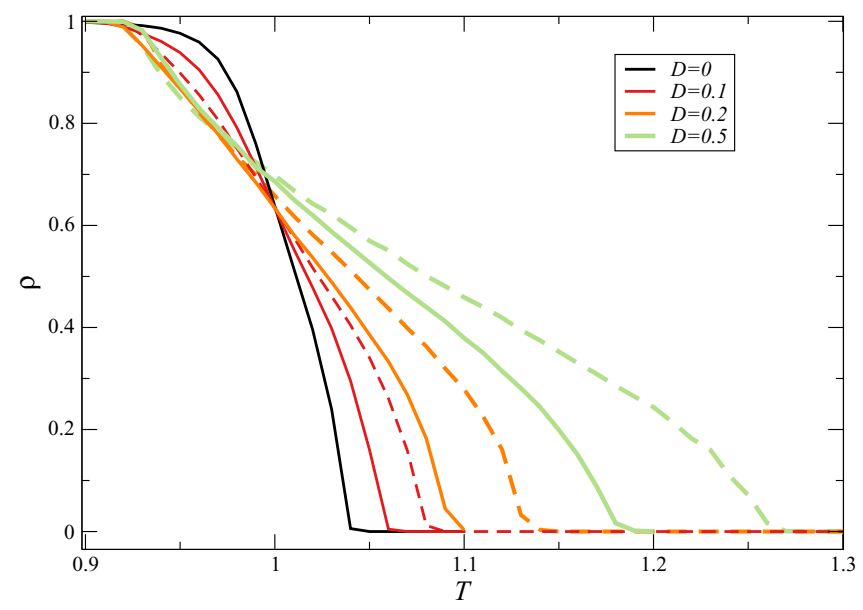

FIG. 4. Average final cooperation level as a function of $T$ for the imitative model comparing the uniform (continuous lines) and Gaussian (dashed lines) distribution. The sets are arranged in pairs with the same $D$ value for the same colors. Higher $D$ values lead to higher survival of the cooperation in the $x$ axis. In the uniform distribution $D$ is the range of the perturbation, while in the normal distribution it is the standard deviation.

control parameter as $D=\sigma$ (i.e., the standard deviation of the distribution). Let us stress that we cannot directly compare the control parameter $D$ for both cases, since in the uniform distribution, $D$ is the range of the distribution, whereas in the Gaussian case, $D$ is the standard deviation. Even so, $D$ relates to the perturbation strength in both scenarios. The results are very similar for the three update rules but with minor quantitative differences, the general difference being that for Gaussian distributions, cooperation is more enhanced than for uniform ones given the same value of $D$. This can be credited to the fact that in a Gaussian distribution with $\sigma=D$, although most perturbations will be equal to or smaller than $D$, there will be rare stronger perturbations occasionally. This, in turn, translates to a more noisy system that can strongly enhance cooperation.

In Fig. 4 we present a comparison between the uniform and Gaussian distributions using the imitative update rule. For the sake of clarity, we present only $D=0.1 ; 0.2 ; 0.5$, but the effect was similar for all ranges of $D \in[0,1]$. As expected (assuming the same control parameter $D$ for both cases), the Gaussian distribution has a more pronounced effect. This suggests that the cooperation enhancement stems from rare and influential fluctuations that are more common in the Gaussian distribution. This is in accordance with a similar hypothesis presented in [55-57].

Considering the imitative rule, we varied the noise level $D$ for different $T$ values. Results are presented in Fig. 5. The perturbation has a positive and continuous effect on the cooperation in regions where $T>1$ and a (small) detrimental effect if $T<1$. Specifically, the strongest cooperation enhancement happens exactly at the phase transition $\left(T_{c}=1.04\right)$. We see that payoff perturbation enhances cooperation for egotistic games $(T>1)$, while it can dampen said cooperation for fraternal games $(T<1)$.

Note that for the unperturbed model, cooperation is extinct for $T>1.04$. However, the resurrection of cooperation after 


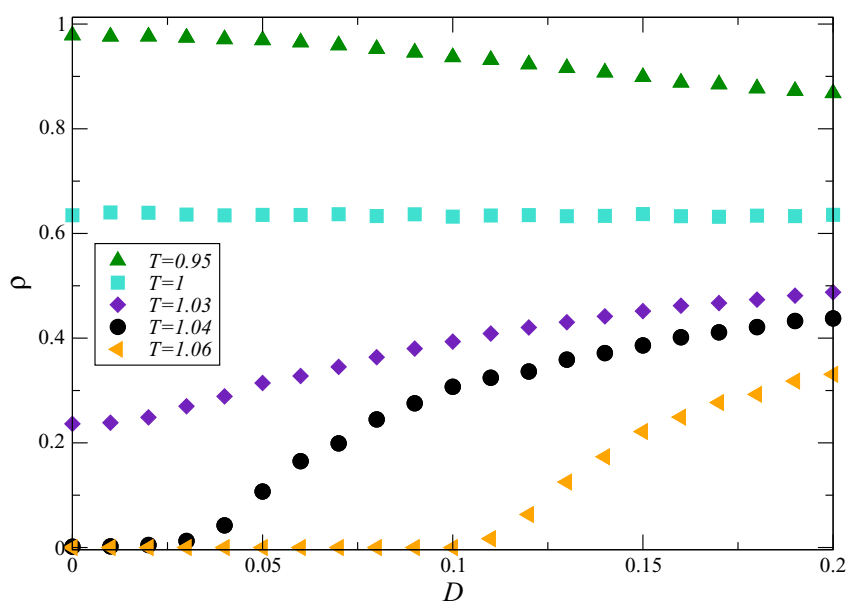

FIG. 5. Average final cooperation level as a function of perturbation strength $D$ for different values of $T$. Black circles represent the critical point $T_{c}=1.04$, were the perturbation effect is stronger.

this critical point is possible given strong enough perturbations. Figure 6 presents the results for the cooperation as a function of $D$ for some cases of $T>T_{c}$. As we can clearly see, there is the separation between an "active" phase with strategy coexistence and a phase where even with perturbation only defectors dominate. The threshold value of $D$ allowing the existence of cooperation, $D_{\text {crit }}$, is shown in the figure inset as a function of $T$. We can see that it behaves mostly linearly with $T$, having an angular coefficient of $\simeq 3.5(5)$.

To understand whether the cooperation boost is caused by any kind of random fluctuation, we compare the effects of payoff perturbation with the noise generated by high irrationality values, $k$, [75,81]. As demonstrated by Szabó et al. [82], irrationality can have beneficial effects on the maintenance of cooperation for some parameter regions. Nevertheless, this is not a linear effect, and there is an "optimal" irrationality level after which the system starts to behave randomly, destroying cooperation. Figure 7 illustrates the final

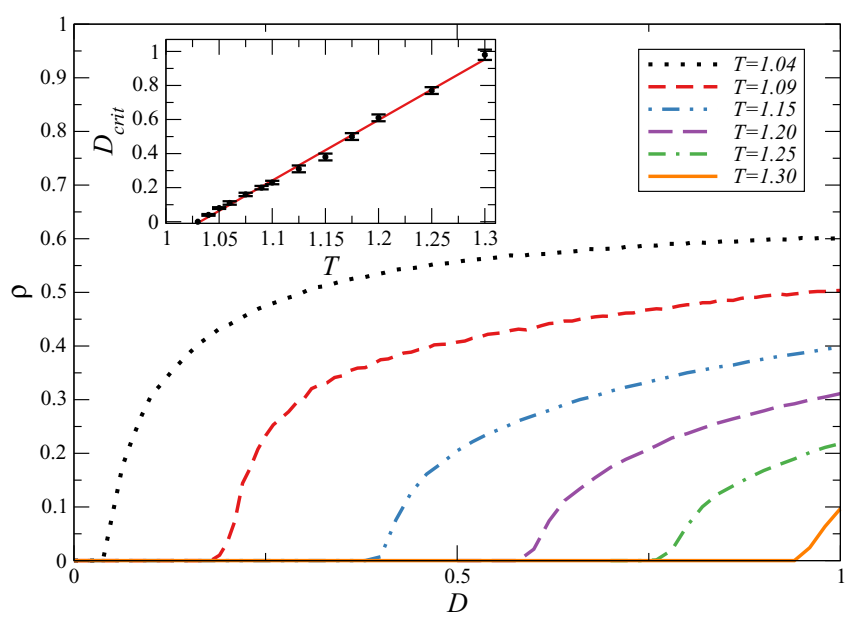

FIG. 6. Average final cooperation level as a function of perturbation strength $D$ for values of $T>T_{c}$, where cooperation is always extinct in the unperturbed model. The inset presents the critical value of $D$, as a function of $T$, that allows cooperation to reemerge after $T_{c}$.

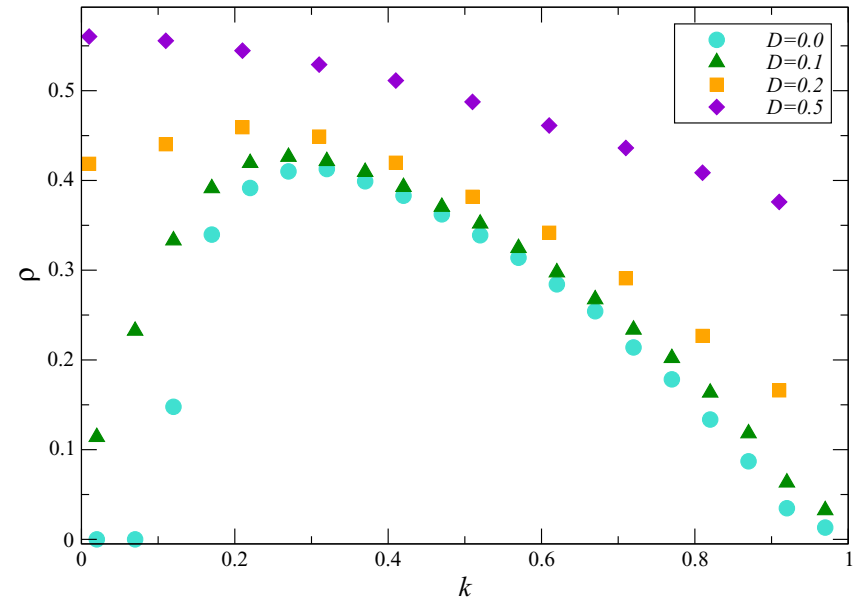

FIG. 7. Average final cooperation level as a function of irrationality $k$ in the phase transition $T=1.04$ for the imitative model. As expected, there is an optimal irrationality level for $D=0$. Even so, payoff fluctuations always increase cooperation for the whole range of explored $k$ values.

average cooperation level as a function of irrationality $k$ for different payoff perturbation strengths. We see that the payoff perturbation boosts cooperation regardless of the irrationality level. Even more, we see that the cooperation enhancement granted by intermediate levels of irrationality is additive with the beneficial effect of the payoff perturbation (more strongly present in the region $0.15<k<0.6$ ). We used $T=1.04$ for the figure, but this effect remained similar for different $T$ values and different update rules in our simulations.

We proceed to analyze which microscopic mechanism is responsible for the cooperation enhancement due to payoff perturbation. It is worth noting that lattice snapshots were not useful in this regard in all three update rule settings. The population has the same general spatial distribution with and without perturbation, i.e., in both cases, cooperators form the usual clusters surrounded by a sea of defectors, the main difference being only that the total fraction of cooperators is greater as we include perturbations. While this does not present the cause of the cooperation enhancement, we see that it is not directly related to a strong spatial distribution effect.

We stress that while the perturbations have zero average value, they can locally change the game class being played at each round. Based on this fact, we analyze how players (locally) fluctuate to a more fraternal or egotistic game. To do so, we define for each player, at each Monte Carlo step, the variable $\phi=\varepsilon_{S}-\varepsilon_{T}$. If $\phi>0(\phi<0)$, the game being played at a given time will be more fraternal (egotistic). Note that for every player, $\bar{\phi}=0$ for long times. Next, we obtain at each time step the population fraction of cooperators and defectors that had $\phi>0$ in the previous round (named fraternal cooperators, $C_{F}$, and fraternal defectors, $D_{F}$ ). We do the same for cooperators and defectors with $\phi<0$ (named egotistic cooperators, $C_{E}$, and egotistic defectors, $D_{E}$ ). By doing so, we can understand if players' strategies are correlated with $\phi$ even if $\varepsilon$ is randomly drawn at every interaction.

Figure 8 presents the average evolution of the four subpopulations for the imitative model in the region where the 


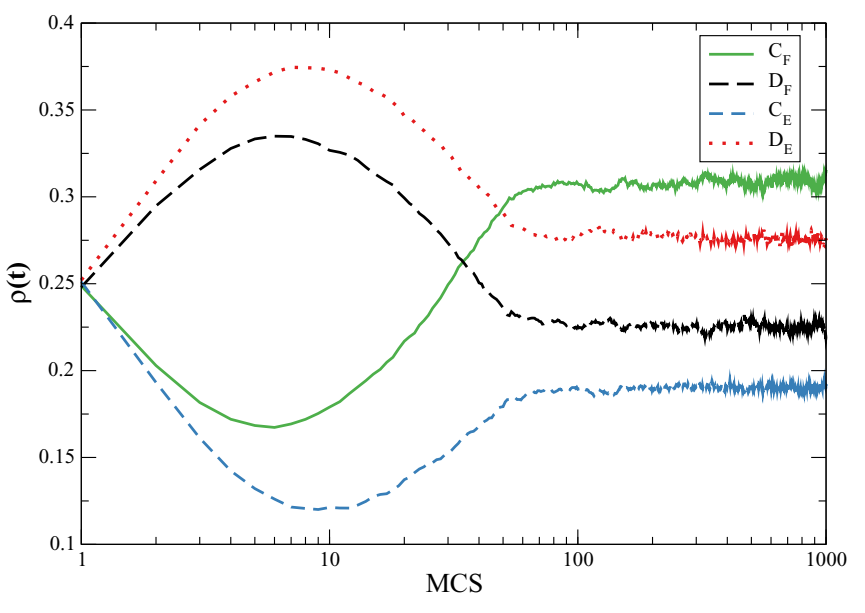

FIG. 8. Average fraction of each subpopulation over 100 Monte Carlos runs. Here we used $D=0.3$ and $T=1.04$, where cooperation would be extinct if not for the payoff perturbation. Fraternal cooperators $\left(C_{F}\right)$ quickly dominate the population, being followed by egotistic defectors $\left(D_{E}\right)$, fraternal defectors $\left(D_{F}\right)$, and lastly, egotistic cooperators $\left(C_{E}\right)$.

perturbation has its strongest effect, i.e., $T_{c}=1.04$. The main effect of payoff perturbation is to separate the subpopulations of players who play, on average, more fraternal or egotistic games. Specifically, fraternal cooperators $\left(C_{F}\right)$ grow more than any other subpopulation, being followed by the egotistic defectors $\left(D_{E}\right)$. It is important to note, however, that even if the perturbations are symmetric, $C_{F}>D_{E}$.

To further explore this, we obtained the fraction of all subpopulations for different $T$ values. The results are shown in Fig. 9. In it we can see that this effect happens for all the relevant $T$ range. On average, sites that have fluctuations leading to $\phi>0$ will tend to become cooperators with

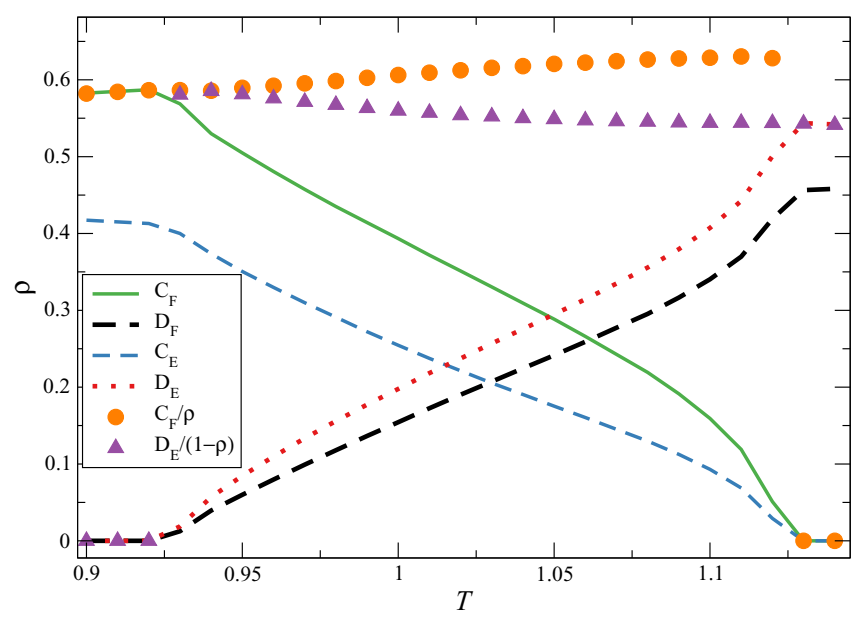

FIG. 9. Average final fraction of each subpopulation (lines) as we vary $T$. Here we used $D=0.3$. Symbols present the average normalized fraction of cooperators that are fraternal, i.e., $C_{F} / \rho$, and the average fraction of defectors that are egotistic, i.e., $D_{E} /(1-\rho)$. Sites that play more fraternal games on average will tend to become cooperators at a higher frequency then egotistic sites will become defectors.

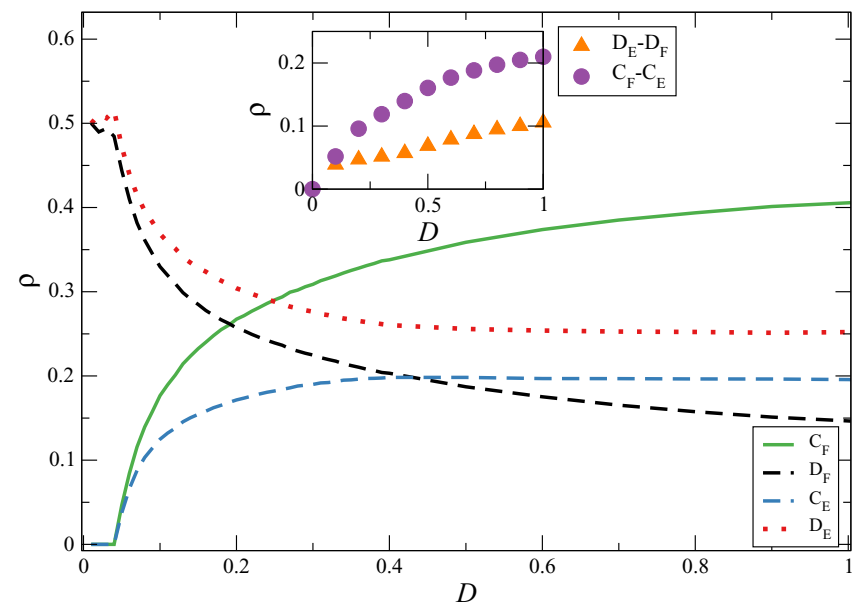

FIG. 10. Average final fraction of each subpopulation (lines) as we vary $D$. Here we used $T=1.04$. The inset shows the difference of the average, fraternal, and egotistic subpopulations $\left(C_{F}-C_{E}\right)$ as well as $\left(D_{E}-D_{F}\right)$.

a higher frequency than sites that had $\phi<0$ will become defectors. Note that the symbols in Fig. 9 present the (normalized) fraction of cooperators that are fraternal, i.e., $C_{F} / \rho$ [and the normalized fraction of defectors that are egotistic, i.e., $\left.D_{E} /(1-\rho)\right]$. This is especially important, since as $T$ increases, the total fraction of cooperators decreases, but even so, $C_{F} / \rho>D_{E} /(1-\rho)$. In other words, even if total cooperation decreases, the perturbation still induces a flux of cooperators to sites where more fraternal games were played. This, in turn, sustains cooperation for greater ranges of $T$.

This analysis was also performed for different perturbation strength values $(D)$ to observe how it can affect the subpopulations. The results are shown in Fig. 10. As expected, the increase in both subpopulations of cooperators is monotonous with $D$. The inset shows the difference $\left(C_{F}-C_{E}\right)$ as well as $\left(D_{E}-D_{F}\right)$. In it we can see that even if a stronger perturbation can lead to a higher value of $\left(D_{E}-D_{F}\right)$, this value is always lower than $\left(C_{F}-C_{E}\right)$. In other words, agents who play more fraternal games on average will have a greater probability of becoming cooperators. On the other hand, the opposite is not true for players that have played, on average, more egotistic games, as their average fraction will be smaller than that of the cooperators. This is the main mechanism behind cooperation enhancement. Simulations for the WSLS and Ising update rules remained similar with small quantitative differences, showing how robust this phenomenon is.

Let us summarize the main mechanism that can increase cooperation in the proposed model; while players have symmetrical perturbations, defectors locally benefit from a more egotistic game but cannot maintain said benefit as their clusters do not obtain benefits from the perturbation $(P$ is not perturbed). At the same time, cooperators can form spatial structures that are more robust against negative perturbations, heaping the long-term benefits of positive perturbations. In other words, cooperators are able to form positive feedback loops with the local positive payoff fluctuations, while defectors cannot. This effect is very similar to what was observed in $[23,83]$, where a very similar positive feedback loop helps 

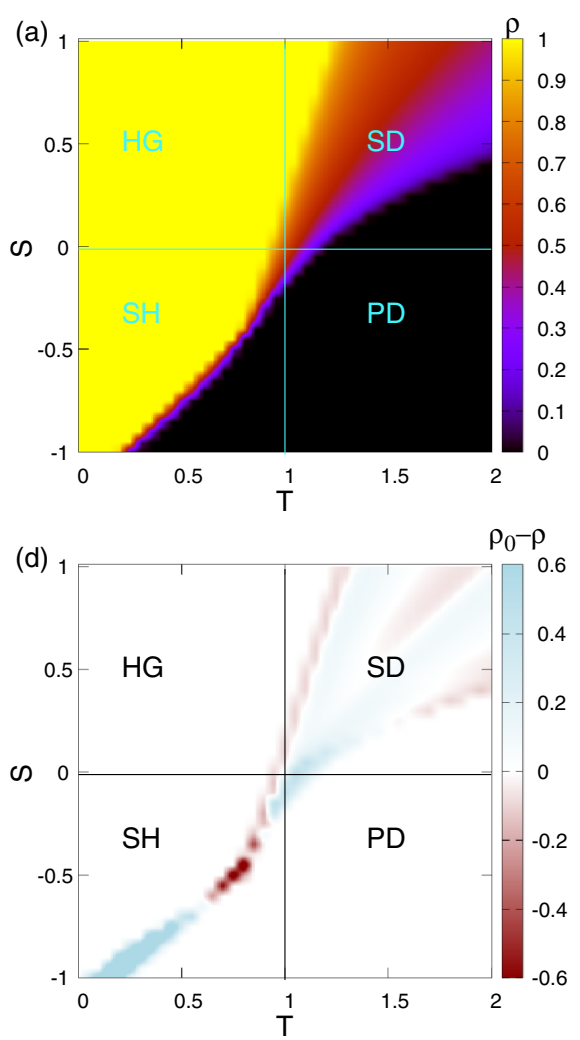
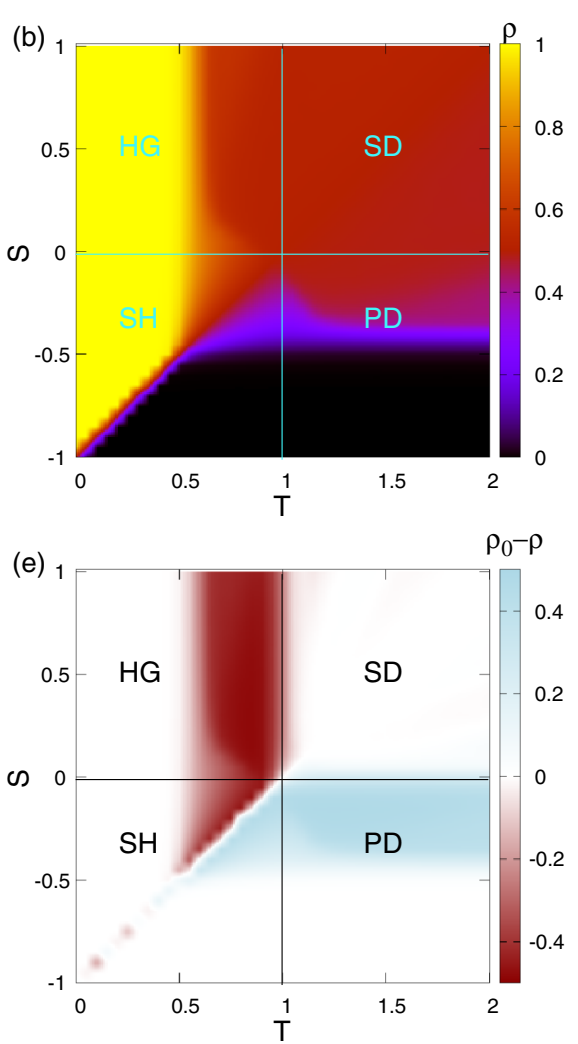
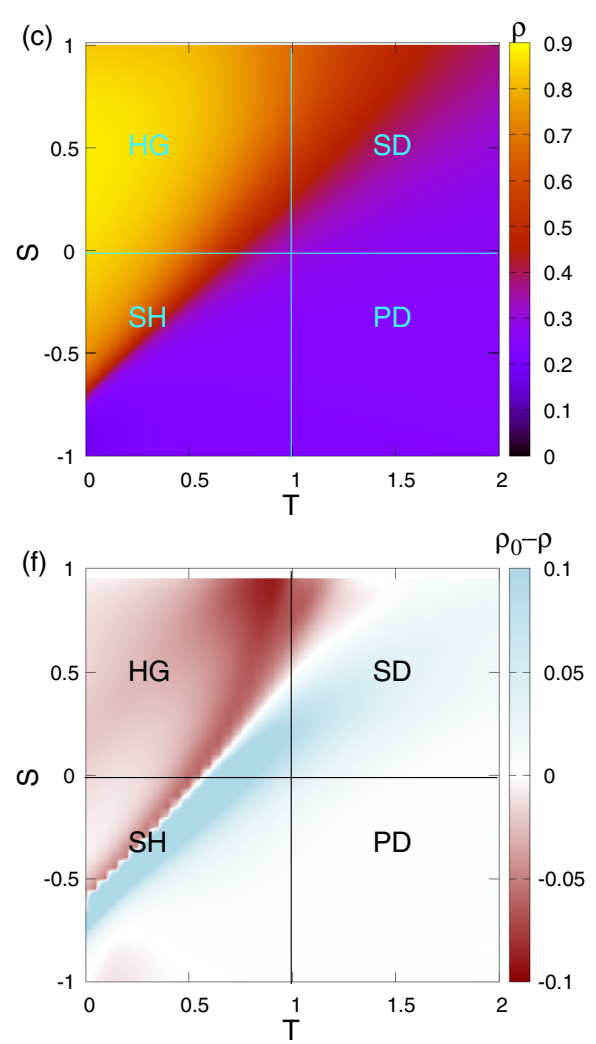

FIG. 11. $T \times S$ parameter space for all studied update rules using a high perturbation strength $D=0.5$. The final cooperation fraction is represented in color in the top row, (a)-(c). The bottom row, (d)-(f), represents the difference $\left(\rho-\rho_{0}\right)$, i.e., the change in cooperation due to perturbation. Dark red represents a negative effect and light blue a positive one. Each column relates to one update model: imitative in (a) and (d), Ising in (b) and (e), and WSLS in (c) and (f). We see that the most general effect of perturbations is to increase cooperation in selfish games while lowering it in altruistic ones. The effects span most of the parameter space but are particularly strong near phase transitions.

the promotion of cooperation for the so-called multigames. Indeed, this seems to be a general property of evolutionary games, i.e., cooperators can benefit from a variety of fluctuations using positive feedback loops whereas defectors cannot.

To conclude, we present the final average cooperation level for the whole parameter space $T \times S$ considering all update rules in Fig. 11. Each update rule is depicted in one column, with the imitative in (a) and (d), Ising in (b) and (e), and WSLS in (c) and (f). The top row depicts the final cooperation fraction in Figs. 11(a)-11(c). The bottom row, Figs. 11(d)11(f), represents the difference $\left(\rho-\rho_{0}\right)$, i.e., the change in cooperation due to perturbation. For all figures, we report the case for a high perturbation $(D=0.5)$. We note that the parameter space for the unperturbed case can be found in the literature in [69] for the win-stay-lose-shift, in [68] for the Ising model, and in [84] for the imitative model.

This analysis indicates that the perturbation changes the population dynamics more strongly near phase transition regions, although it is not restricted only to this region of the parameter space. By using all three updating rules, we can observe the general effect that perturbation increases cooperation in the direction of more selfish games (i.e., high $T$, low $S$ ), while it does the opposite for altruistic games. We also note that the detrimental effect on cooperation is usually lower than the benefit for regions with high $T$ and a low $S$. We deem it especially interesting that by using the Ising rule, see Figs. 11(b) and 11(e), the perturbation increases the anticoordination area linearly with the perturbation strength (top right region of the $T \times S$ parameter space).

\section{CONCLUSIONS}

In this work, we studied payoff perturbations considering simple social dilemmas, with the main goal to clarify and quantify their effects when only the off-diagonal is interested, following the results reported in [8]. Beyond analyzing perturbative methods in evolutionary games, the proposed model has the potential to actively contribute to a vivid debate, i.e., that on the relationship between heterogeneity and cooperation. We emphasize that the form of heterogeneity we consider is related to that of risk and reward perception, i.e., an aspect covering a fundamental role in many social systems and that might also be relevant, using a different interpretation, in other contexts.

Then, while [8] focused on a general range of effects resulting from perturbed payoffs, here we concentrate on the strategy equilibrium reached by a population in the presence of perturbations acting only on the rewards of betraying and the risks involved in getting betrayed (i.e., the temptation and the sucker's payoff). Hence, cooperators playing with cooperators, and defectors playing with defectors, are not affected by perturbations. In addition, here we analyze a wider range of game parameters, i.e., near and far phase transition points, 
in order to identify those values that allow perturbations to actively affect the dynamics of the game.

The evolution of strategies has been studied by Monte Carlo simulations, arranging players on a square lattice with periodic boundary conditions. To verify if the observed effects were robust in different settings, we considered three different update rules-imitation, win-stay-lose-shift, and Ising. We also analyzed the effects of Gaussian and uniform noise distributions.

Results show that perturbations can have more pronounced effects on the population dynamics, mainly near the phase transitions. Even so, such perturbations can have effects on games far away from the phase transition region, although generating a weaker effect. Notably, the average evolution of a population showed that the perturbations act very quickly, affecting mainly the initial evolution. Also, this effect is proportional to the perturbation amplitude when $T>1$. At the same time, the dampening in cooperation observed for $T<1$ seems to be very small in comparison. In addition, the overall effect of the perturbation seems to be independent of the specific choice of the update rule, highlighting its robustness. Then the analysis related to the combined effect of heterogeneity and irrationality showed that these two perturbation sources are quite independent of each other.

In order to understand the microscopic mechanism responsible for the cooperation enhancement near phase transitions, we studied the subpopulation of cooperators and defectors that, locally, played more fraternal or egotistic games at each time step. This is in line with previous works which proposed that the benefit of diversity would be related to the random changes in the game class (prisoner's dilemma changing to stag hunt or snowdrift) [55-57]. This approach allowed us to unveil what was responsible for the general effect observed in all cases. On average, we found that the payoff perturbation offers no unilateral contribution for more egotistic fraternal games. Even so, it can locally promote cooperation for players that had a more positive perturbation. This effect can lead to the formation of stronger cooperative clusters, while defectors cannot benefit from said phenomena in the long run, as they do not benefit from mutual support (see also [63]). On the other hand, in [58] authors presented arguments on the irrelevance of game class changes for some contexts. While this seems to be an open topic, our initial results indicate that the random fluctuations have deep effects on the phase transitions of the model, being able to improve cooperation thanks to the unusual states found near phase transition points.

Summarizing, our main finding is that off-diagonal perturbations cannot be considered as a trivial form of noise, as they seem to selectively enhance cooperation when defection is pervading the population, while they are able to support defection as the population begins to cooperate. Therefore, in our view the proposed model offers interesting insights on a relevant aspect of heterogeneity, not limited only to the study of social phenomena. Notwithstanding, since several details might deserve further attention, we report those we consider potentially more interesting. For instance, the proposed model could be analyzed considering populations arranged on complex networks $[85,86]$, social behaviors like conformism [87-89] could be combined with random perturbations, and as suggested in [60], also some clinical investigations could find it useful to represent specific biological phenomena by the proposed method.

Finally, we see as especially interesting the connection of the payoff perturbation with recent works connecting evolutionary game theory with the Hamiltonian description of physical systems $[84,90]$. These effects could be further studied in light of temporal Griffiths phases [67,91]. Such an exotic state is known to appear near phase transitions in epidemiological and magnetic systems with a directed percolation universality class, which is the same phase transition as the imitative model [51].

\section{ACKNOWLEDGMENT}

This research was supported by the Brazilian Research Agency CNPq (Project No. 428653/2018-9).
[1] H. Broer and F. Takens, Dynamical Systems and Chaos (Springer, New York, 2010).

[2] B. Bransden and C. Joachain, Quantum Mechanics (Pearson, Harlow, UK, 2000).

[3] P. Smith, Explaining Chaos (Cambridge University Press, Cambridge, UK, 1998).

[4] "The Oxford Murders" (2008), directed by Alex de la Iglesia, adapted from the novel of the same name by mathematician and writer Guillermo Martinez.

[5] V. Capraro and M. Perc, Front. Phys. 6, 107 (2018).

[6] C. Castellano, S. Fortunato, and V. Loreto, Rev. Mod. Phys. 81, 591 (2009).

[7] E. H. Hagen and P. Hammerstein, Theor. Popul. Biol. 69, 339 (2006).

[8] M. A. Amaral and M. A. Javarone, Proc. R. Soc. A 476, 20200116 (2020).
[9] J. M. Smith, Evolution and the Theory of Games (Cambridge University Press, Cambridge, UK, 1982).

[10] M. A. Nowak, Evolutionary Dynamics Exploring the Equations of Life (Harvard University Press, Cambridge, MA, 2006).

[11] M. Perc, J. J. Jordan, D. G. Rand, Z. Wang, S. Boccaletti, and A. Szolnoki, Phys. Rep. 687, 1 (2017).

[12] E. Pennisi, Science 309, 93 (2005).

[13] W. Hamilton, J. Theor. Biol. 7, 17 (1964).

[14] R. L. Trivers, Q. Rev. Biol. 46, 35 (1971).

[15] R. Axelrod and W. Hamilton, Science 211, 1390 (1981).

[16] M. A. Nowak and R. M. May, Nature (London) 359, 826 (1992).

[17] S. Nag Chowdhury, S. Kundu, M. Duh, M. Perc, and D. Ghosh, Entropy 22, 485 (2020).

[18] L. Wardil and J. K. L. da Silva, Europhys. Lett. 86, 38001 (2009). 
[19] L. Wardil and J. K. L. da Silva, Phys. Rev. E 81, 036115 (2010).

[20] D. S. Wilson, Am. Nat. 111, 157 (1977).

[21] M. Perc and A. Szolnoki, Biosystems 99, 109 (2010).

[22] A. Szolnoki and Z. Danku, Physica A 511, 371 (2018).

[23] M. A. Amaral, L. Wardil, M. Perc, and J. K. L. da Silva, Phys. Rev. E 93, 042304 (2016).

[24] M. A. Amaral, L. Wardil, and J. K. L. da Silva, J. Phys. A Math. Theor. 48, 445002 (2015).

[25] J. Tanimoto, Appl. Math. Comput. 304, 20 (2017).

[26] F. C. Santos, F. L. Pinheiro, T. Lenaerts, and J. M. Pacheco, J. Theor. Biol. 299, 88 (2012).

[27] Z. Wang, A. Szolnoki, and M. Perc, Phys. Rev. E 90, 032813 (2014).

[28] A. J. Stewart, T. L. Parsons, and J. B. Plotkin, Proc. Natl. Acad. Sci. USA 113, E7003 (2016).

[29] J. Qin, Y. Chen, Y. Kang, and M. Perc, Europhys. Lett. 118, 18002 (2017).

[30] X.-D. Zhang, Z. Wang, F.-F. Zheng, and M. Yang, Chin. Phys. B 21, 030205 (2012).

[31] M. R. Arefin, T. Masaki, K. M. A. Kabir, and J. Tanimoto, Proc. R. Soc. London, Ser. A 475, 20190608 (2019).

[32] J. H. Holland, Sci. Am. 267, 66 (1992).

[33] A. Szolnoki and M. Perc, Sci. Rep. 9, 12575 (2019).

[34] H. Takesue, Europhys. Lett. 126, 58001 (2019).

[35] M. A. Amaral and M. A. Javarone, Phys. Rev. E 97, 042305 (2018).

[36] L. Zhou, B. Wu, V. V. Vasconcelos, and L. Wang, Phys. Rev. E 98, 062124 (2018).

[37] R. P. Mann and D. Helbing, Proc. Natl. Acad. Sci. USA 114, 5077 (2017).

[38] M. Perc and A. Szolnoki, Phys. Rev. E 77, 011904 (2008).

[39] C. Gracia-Lazaro, A. Ferrer, G. Ruiz, A. Tarancon, J. A. Cuesta, A. Sanchez, and Y. Moreno, Proc. Natl. Acad. Sci. USA 109, 12922 (2012).

[40] M. A. Javarone and D. Marinazzo, PLoS ONE 12, e0187960 (2017).

[41] A. Antonioni, L. A. Martinez-Vaquero, C. Mathis, L. Peel, and M. Stella, Phys. Rev. E 99, 052311 (2019).

[42] M. H. Duong, H. M. Tran, and T. A. Han, J. Math. Biol. 78, 331 (2019).

[43] R. L. Shuler, Heliyon 5, e02958 (2019).

[44] R. Lawton, O. Robinson, R. Harrison, S. Mason, M. Conner, and B. Wilson, BMJ Qual. Saf. 28, 382 (2019).

[45] M. A. Javarone, Europhys. Lett. 110, 58003 (2015).

[46] J. Vicens, N. Bueno-Guerra, M. Gutiérrez-Roig, C. GraciaLázaro, J. Gómez-Gardeñes, J. Perelló, A. Sánchez, Y. Moreno, and J. Duch, PLoS ONE 13, e0204369 (2018).

[47] Y. Fang, T. P. Benko, M. Perc, and H. Xu, Sci. Rep. 9, 7655 (2019).

[48] X. Liu, Q. Pan, M. He, and A. Liu, Physica A 521, 258 (2019).

[49] Z. Deng, M. Deming, and D. Dameng, Chaos Solitons Fractals 107, 88 (2018).

[50] E. J. Júnior, M. A. Amaral, and L. Wardil, Physica A 540, 123195 (2020).

[51] G. Szabó and G. Fáth, Phys. Rep. 446, 97 (2007).
[52] Q. Su, A. McAvoy, L. Wang, and M. A. Nowak, Proc. Natl. Acad. Sci. USA 116, 25398 (2019).

[53] G.-Q. Zhang, Q.-B. Sun, and L. Wang, Chaos Solitons Fractals 51, 31 (2013).

[54] M. Perc, New J. Phys. 13, 123027 (2011).

[55] M. Perc, New J. Phys. 8, 183 (2006).

[56] M. Perc, New J. Phys. 8, 22 (2006).

[57] M. Perc, Europhys. Lett. 75, 841 (2006).

[58] J. Tanimoto, Phys. Rev. E 76, 041130 (2007).

[59] J. Hofbauer and W. H. Sandholm, J. Econ. Theory 132, 47 (2007).

[60] F. Stollmeier and J. Nagler, Phys. Rev. Lett. 120, 058101 (2018).

[61] M. Alam, K. Nagashima, and J. Tanimoto, Chaos Solitons Fractals 114, 338 (2018).

[62] C. Hilbe, Š. Šimsa, K. Chatterjee, and M. A. Nowak, Nature (London) 559, 246 (2018).

[63] M. A. Javarone, Eur. Phys. J. B 89, 42 (2016).

[64] J. Tanimoto, Physica A 462, 714 (2016).

[65] T. Yakushkina, D. B. Saakian, A. Bratus, and C.-K. Hu, J. Phys. Soc. Japan 84, 064802 (2015).

[66] T. A. Han, A. Traulsen, and C. S. Gokhale, Theor. Popul. Biol. 81, 264 (2012).

[67] C. E. Fiore, M. M. de Oliveira, and J. A. Hoyos, Phys. Rev. E 98, 032129 (2018).

[68] M. A. Amaral, M. Perc, L. Wardil, A. Szolnoki, E. J. da Silva Júnior, and J. K. L. da Silva, Phys. Rev. E 95, 032307 (2017).

[69] M. A. Amaral, L. Wardil, M. Perc, and J. K. L. da Silva, Phys. Rev. E 94, 032317 (2016).

[70] Z. Danku, Z. Wang, and A. Szolnoki, Europhys. Lett. 121, 18002 (2018).

[71] F. Dercole, F. Della Rossa, and C. Piccardi, Sci. Rep. 9, 5367 (2019).

[72] R. J. Glauber, J. Math. Phys. 4, 294 (1963).

[73] G. Szabó, A. Szolnoki, and L. Czakó, J. Theor. Biol. 317, 126 (2013).

[74] K. Binder and D. P. Landau, Phys. Rev. B 21, 1941 (1980).

[75] M. A. Javarone and F. Battiston, J. Stat. Mech.: Theory Exp. (2016) 073404.

[76] E. Bonawitz, S. Denison, A. Gopnik, and T. L. Griffiths, Cogn. Psychol. 74, 35 (2014).

[77] M. Perc and Z. Wang, PLoS ONE 5, e15117 (2010).

[78] J. M. Pacheco, A. Traulsen, and M. A. Nowak, Phys. Rev. Lett. 97, 258103 (2006).

[79] W. Chen, T. Wu, Z. Li, and L. Wang, J. Stat. Mech.: Theory Exp. (2015) P01032.

[80] D. P. Landau and K. Binder, A Guide to Monte Carlo Simulations in Statistical Physics (Cambridge University Press, Cambridge, UK, 2000).

[81] J. Vukov, G. Szabó, and A. Szolnoki, Phys. Rev. E 73, 067103 (2006).

[82] G. Szabó, J. Vukov, and A. Szolnoki, Phys. Rev. E 72, 047107 (2005).

[83] A. Szolnoki and M. Perc, Europhys. Lett. 108, 28004 (2014).

[84] G. Szabó and I. Borsos, Phys. Rep. 624, 1 (2016). 
[85] Y. Wu, S. Chang, Z. Zhang, and Z. Deng, Sci. Rep. 7, 41076 (2017).

[86] F. Battiston, M. Perc, and V. Latora, New J. Phys. 19, 073017 (2017).

[87] S. Galam, Int. J. Mod. Phys. C 19, 409 (2008).

[88] S. Galam, Physica A 333, 453 (2004).
[89] M. A. Javarone, A. Antonioni, and F. Caravelli, Europhys. Lett. 114, 38001 (2016).

[90] G. Nakamura, G. Contesini, and A. Martinez, Physica A 515, 102 (2019).

[91] F. Vazquez, J. A. Bonachela, C. López, and M. A. Muñoz, Phys. Rev. Lett. 106, 235702 (2011). 